10

\title{
Акустооптическое управление энергетическим 2D-профилем лазерного луча
}

\author{
(C) С.Н. Антонов, ${ }^{1}$ Ю.Г. Резвов ${ }^{2}$ \\ ${ }^{1}$ Фрязинский филиал Института радиотехники и электроники им. В.А. Котельникова РАН, \\ 141190 Фрязино Московская обл., Россия \\ ${ }^{2}$ Новомосковский институт Российского химико-технологического университета им. Д.И. Менделеева, \\ 301665, Новомосковск Тульская обл., Россия \\ e-mail: olga-ant@yandex.ru, rezvovyug@mail.ru
}

Поступило в Редакцию 26 января 2021 г.

В окончательной редакции 1 марта 2021 г.

Принято к публикации 3 марта 2021 г.

Рассмотрено акустооптическое управление энергетическим профилем лазерного излучения. Использован высокоэффективный многолучевой режим брэгговской дифракции с комбинацией близких в угловом пространстве лучей, формирующих усредненную картину в виде единого луча. При этом двумерный профиль интенсивности реализован в виде произведения двух независимых одномерных профилей. На базе поляризационно-независимого двухкоординатного акустооптического дефлектора экспериментально получены несколько профилей (в том числе близкий к равномерному) при суммарной эффективности не ниже $85 \%$ и времени смены профиля около $10 \mu \mathrm{s}$. Данный метод может быть использован в системах обработки материалов мощными лазерами.

Ключевые слова: лазер, поляризация света, анизотропная акустооптическая дифракция, акустооптический дефлектор, пьезопреобразователь, эффективность дифракции, энергетический профиль лазерного излучения.

DOI: 10.21883/JTF.2021.08.51104.14-21

\section{Введение}

Создание мощных промышленных лазеров привело к их широкому использованию для обработки материалов - резки, плавления, нанесения изображений и т.д. [1-4] Поперечное распределение интенсивности излучения в лазерном пучке зависит от набора мод, характерных для используемого резонатора. В частности, в одномодовом случае оно близко к распределению Гаусса (рис. 1, $a): I(x, y) \propto \exp \left(-2\left(r / r_{0}\right)^{2}\right)$, $r^{2}=\left(x^{2}+y^{2}\right), r_{0}-$ радиус пучка по уровню $1 / e^{2}$. При обработке материалов такое распределение не является оптимальным, так как воздействие обычно имеет пороговый характер и та часть излучения, интенсивность которой ниже порога, не используется. Кроме того, технологически более приемлемая форма светового пятна может не иметь аксиальной симметрии. На рис. $1, b-d$ представлены практически используемые распределения интенсивности лазерного луча на целевой плоскости. В некоторых случаях распределение должно быть максимально равномерным с четко очерченными границами (рис. $1, b, c)$. Иногда оптимальным является распределение с провалом интенсивности в центре картины (рис. $1, d$ ). Во всех четырех случаях мощность излучения одинакова, поэтому максимальная интенсивность в уширенных негауссовых пятнах меньше, чем в центре гауссового (где принята за единицу).

Для изменения поперечного распределения интенсивности лазерного излучения (beam shaping) используются различные технологии: рефракционная оптика, решетки микролинз и микрозеркал, пространственные модуляторы света [5-7]. Рис. 2 - пример промышленной модели шейпера, в данном случае линзовой системы [8], трансформирующей гауссов лазерный луч в излучение с неизменяемым профилем, близким к прямоугольному.

В системах лазерной обработки материалов используются в том числе акустооптические технологии. Прикладная акустооптика (AO) основана на фотоупругом эффекте, возникающем при распространении ультразвука в прозрачных средах, и направлена на управление параметрами оптического излучения [9-15]. Принципиальными особенностями АО приборов являются: возможность управления интенсивным лазерным излучением в десятки и сотни $\mathrm{kW} / \mathrm{cm}^{2}$, высокое быстродействие (до десятков наносекунд), отсутствие механически перемещаемых элементов, малые вносимые световые потери (единицы процентов), небольшие габариты и вес.

Перспективно применение АО технологий для управляемого формирования поперечного профиля интенсивности излучения [16-20]. В частности, в недавних работах $[21,22]$ для одномерной и двумерной трансформации профиля используются пространственно-частотные фильтрующие свойства АО взаимодействия. В работах $[23,24]$ представлено формирование одномерного профиля за счет сложения нескольких лучей, полученных посредством АО дифракции на многочастотном электрическом сигнале. Настоящая работа развивает метод, предложенный в [23], и представляет поляризационно-независимый двухкоординатный быст- 

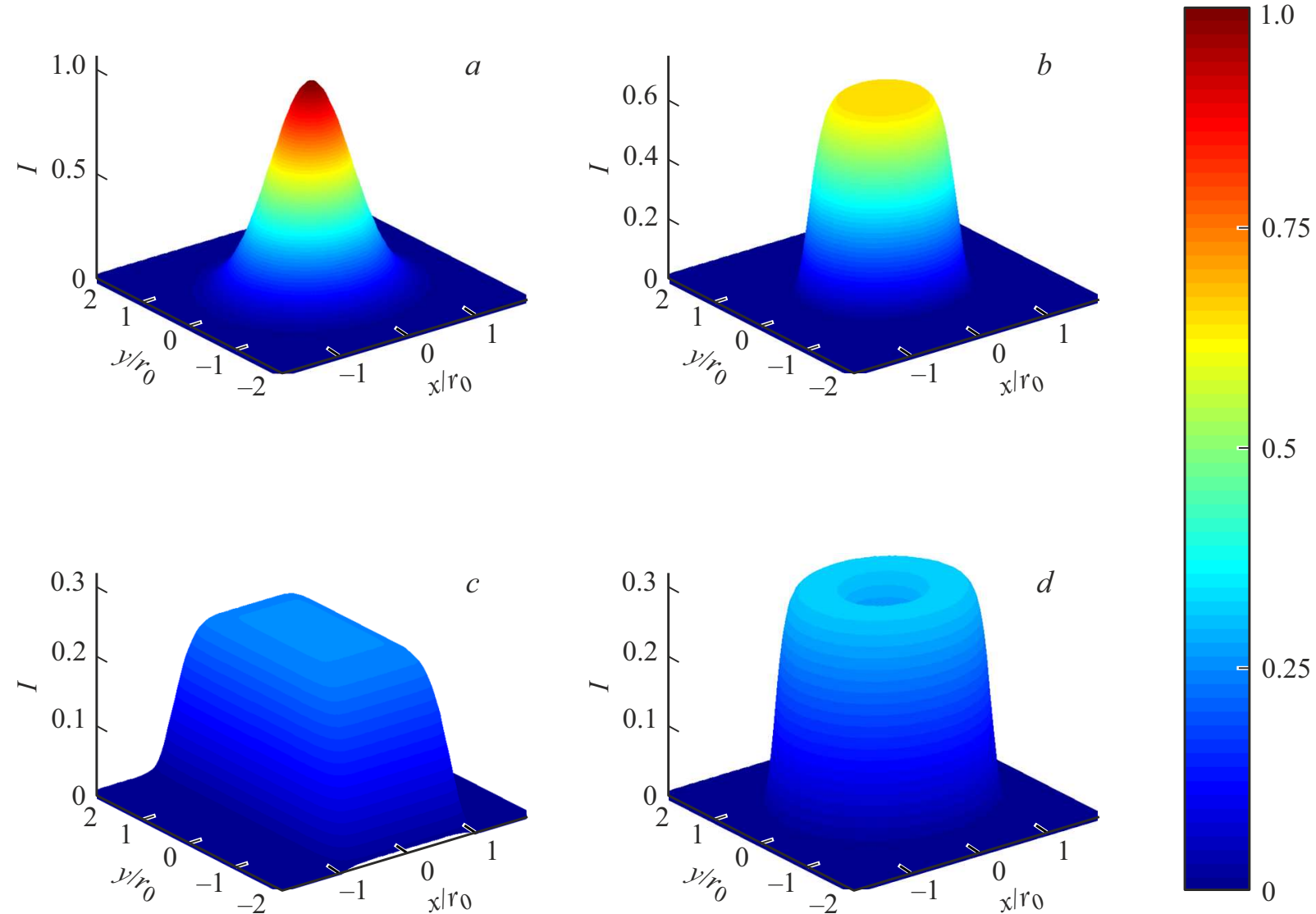

Рис. 1. Поперечное распределение интенсивности лазерного пятна на целевой плоскости: гауссово $(a)$, близкое к равномерному $(b, c)$, аксиально-симметричное с провалом в центре $(d)$.
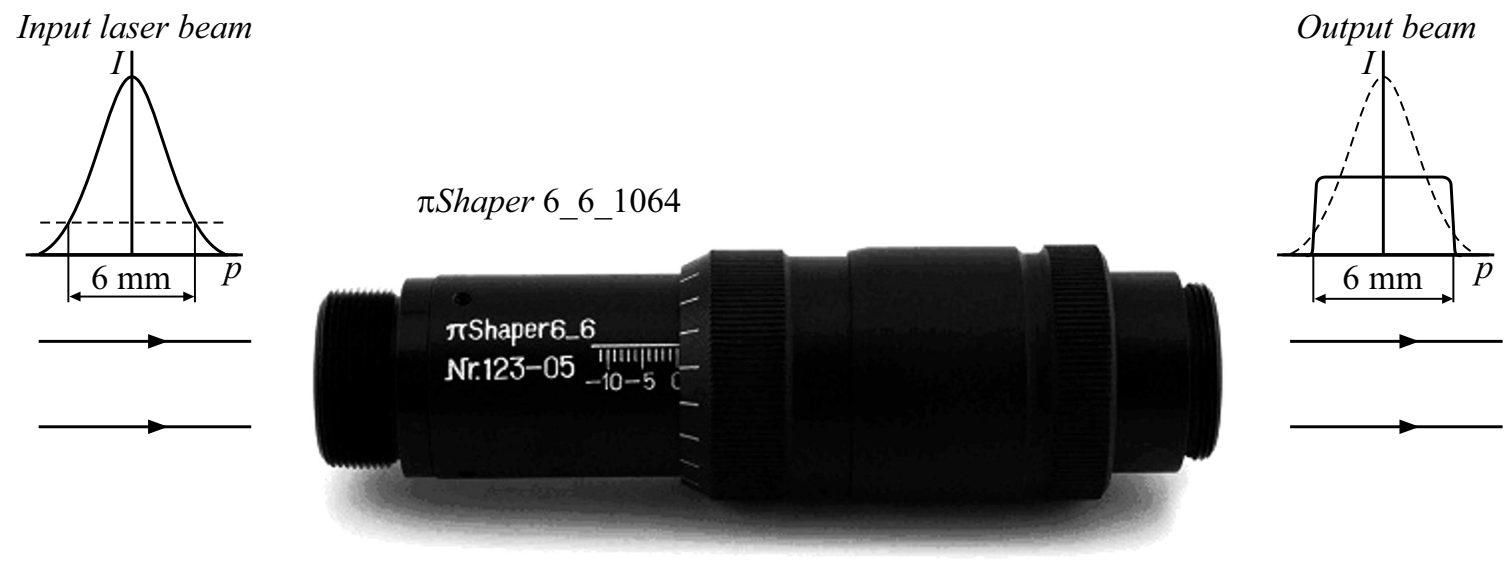

Рис. 2. Прибор серии $\pi$ Shaper.

ро перестраиваемый $\mathrm{AO}$ шейпер, параметры которого управляются только выбором радиосигнала.

\section{1. Методы}

Принцип работы двухкоординатного перестраиваемого шейпера базируется на режиме многолучевой брэгговской дифракции. Практическая реализация основана на использовании поляризационно-независимо двухкоординатного АО дефлектора на базе кристалла парателлурита $\left(\mathrm{TeO}_{2}\right)$.

\section{1. Многолучевая акустооптическая дифракция}

Пусть на АО ячейку подан радиосигнал, образованный дискретным набором монохроматических составляющих 
с частотами $f_{1}, f_{2}, f_{3}, \ldots$ При малом уровне сигнала дифрагированное поле содержит дискретный ряд субпорядков, волновые векторы которых $\left(\mathbf{k}_{d 1}, \mathbf{k}_{d 2}, \mathbf{k}_{d 3}, \ldots\right)$ однозначно соответствуют частотам радиосигнала (рис. 3 ). Угол отклонения каждого субпорядка от проходящего света (единственный субпорядок с волновым вектором $\mathbf{k}_{t 0}$ ) пропорционален частоте отдельной компоненты радиосигнала: $\Theta_{1} \sim f_{1}$ и т.д. При увеличении уровня сигнала вследствие нелинейного характера $\mathrm{AO}$ взаимодействия проходящий и дифрагированный свет обогащаются интермодуляционными составляющими (наборы $\mathbf{k}_{t, i m}$ и $\mathbf{k}_{d, i m}$ ). При этом мощность каждого из основных и интермодуляционных субпорядков нелинейным образом зависит от амплитуд и фаз частотных компонент радиосигнала. Результатом интермодуляционных процессов становится потеря лазерной мощности и снижение контраста в дифрагированном поле.

Однако было экспериментально обнаружено, что в некоторых случаях многочастотный характер акустического сигнала не вызывает существенных интермодуляционных искажений. Исследование этого явления позволило с малыми потерями делить падающий лазерный луч АО методами $[25,26]$.

Установлено, что интермодуляционные эффекты минимальны, если акустическая волна имеет минимальные амплитудные искажения, т.е. для формирования высокоэффективного многолучевого поля с точным соответствием между частотным спектром радиосигнала и пространственным спектром многолучевого поля акустическая волна должна иметь угловую (частотную, фазовую) модуляцию. Чтобы дифрагированное многолучевое поле было практически стационарным, необходима периодическая фазовая модуляция акустической волны, причем в каждый момент на световой апертуре должно „умещаться" несколько пространственных периодов модуляции (рис. 4).

Здесь $\Delta \varphi_{s}$ - периодическое с периодом $T$ изменение фазы акустической волны, что дает пространственный период модуляции, равный $v_{s} T$, где $v_{s}$ - скорость звука. Видно, что на световой апертуре диаметром $2 r_{0}$ умещается несколько пространственных периодов. Это

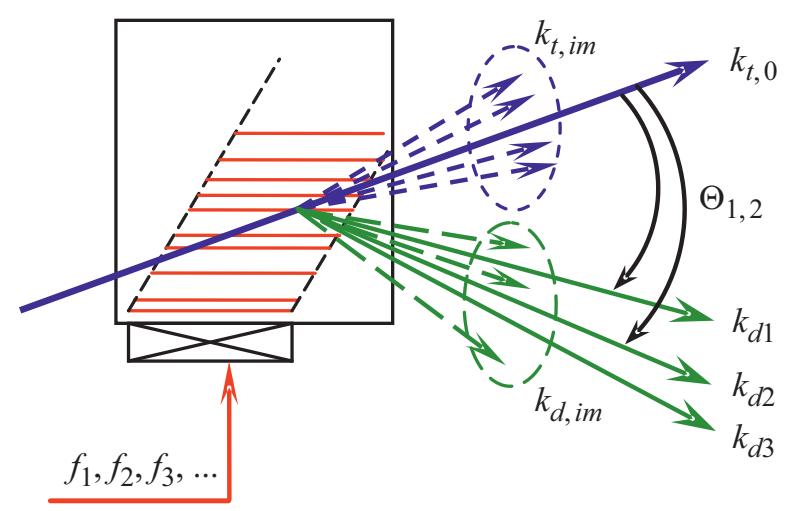

Рис. 3. Структура проходящего и дифрагированного поля при использовании многочастотного радиосигнала.

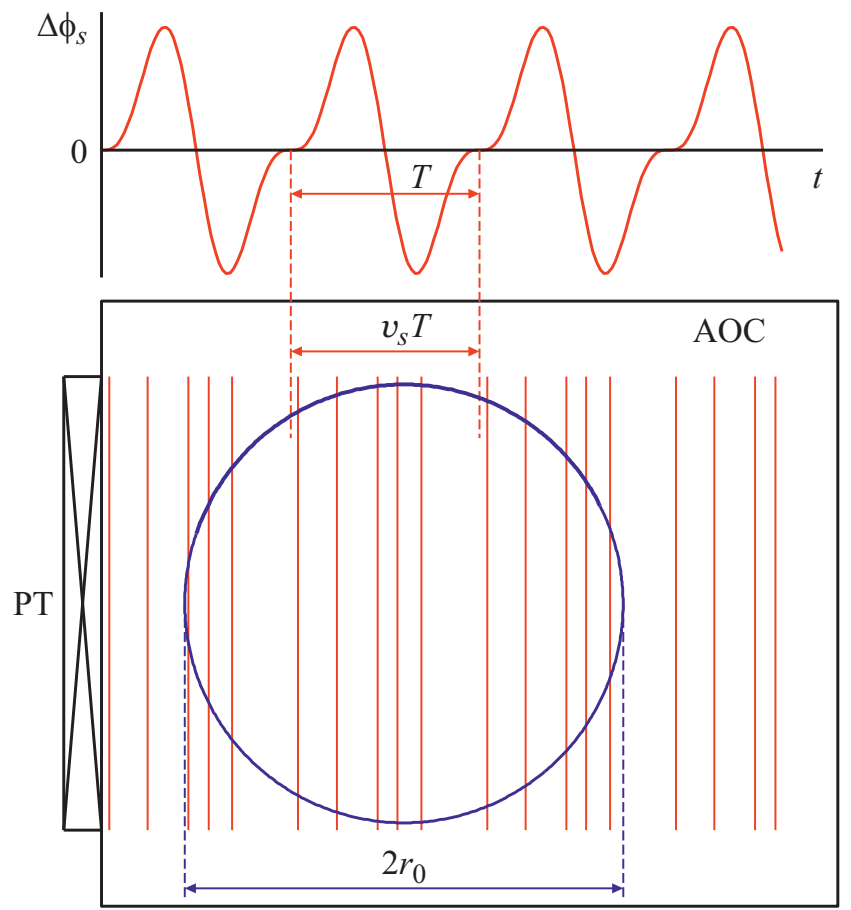

Рис. 4. Иллюстрация формирования стационарного дифракционного поля без интермодуляционных искажений. АОС - $\mathrm{AO}$ ячейка, РТ - пьезопреобразователь.

условие необходимо, так как при брэгговской АО дифракции частота колебаний в каждом субпорядке дифрагированного поля смещена на соответствующую частоту звука относительно падающего света. В результате при частичном наложении субпорядков возникает модуляция интенсивности. Указанное условие „разводит“ субпорядки, поэтому распределение световой мощности по дифрагированным лучам точно отражает распределение мощности по частотным компонентам акустического сигнала.

Для формирования заданного многолучевого дифракционного поля был разработан метод цифрового синтеза сигнала из конечного набора монохроматических частотных компонент. Для узкополосных сигналов необходимо подобрать такие фазы компонент, чтобы результирующий сигнал максимально приблизился к оптимальному фазово-модулированному виду. При этом фазы составляющих не являются жестко определенными, а подчиняются связям, число которых меньше числа частот. При увеличении полосы частот необходима коррекция сигнала, что позволяет дополнительно увеличить полезную эффективность.

Рассмотрим модуляцию интенсивности при частичном наложении субпорядков на примере сигнала, сформированного из 7 эквидистантных частотных компонент: $f_{0}$, $f_{0} \pm \Delta f, f_{0} \pm 2 \Delta f, f_{0} \pm 3 \Delta f$. Для гауссова пучка с распределением в перетяжке вида $I(x, y) \propto \exp \left(-2\left(r / r_{0}\right)^{2}\right)$ угловой спектр интенсивности имеет аналогичный вид: $I \propto \exp \left(-2\left(\theta / \theta_{0}\right)^{2}\right)$, где $\theta_{0}=\lambda / \pi r_{0}-$ угол между цен- 


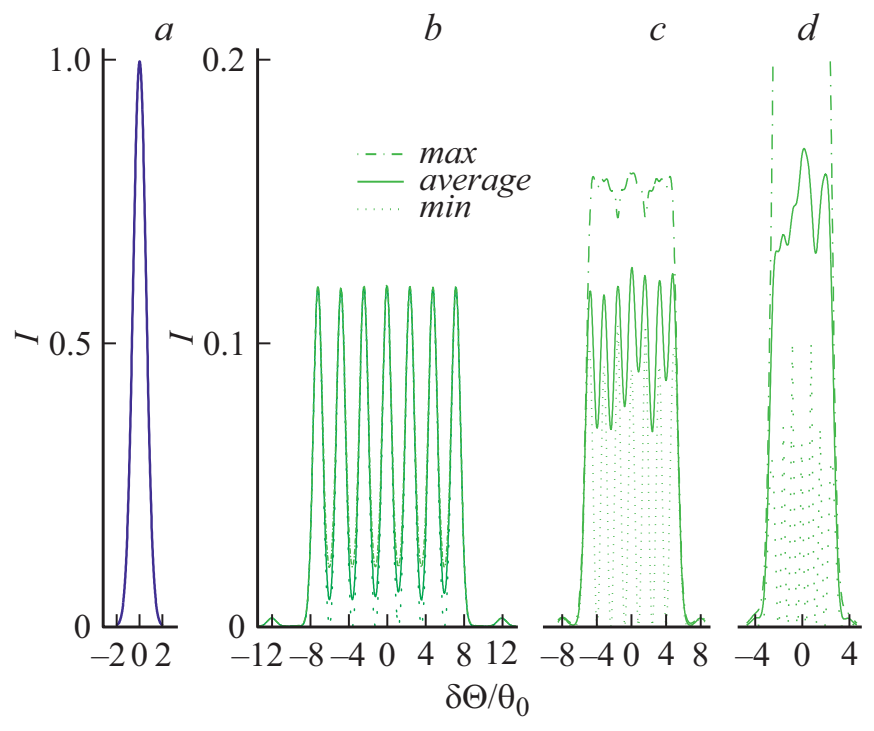

Рис. 5. Центрированное угловое распределение интенсивности падающего света $(a)$ и дифракционного поля $(b-d)$ при $\Delta \Theta / \theta_{0}=2.5(b), 1.5(c), 1.0(d)$. Угол $\delta \Theta=\Theta-\Theta_{0}$ отсчитывается от центра картины.

тральной волной и направлением, при условии, что интенсивность уменьшена в $e^{2}$ раз. Оптимизация фаз компонент приближает акустический сигнал к фазовомодулированному, в результате можно сформировать высокоэффективное дифракционное семи лучевое поле. Профиль каждого луча почти совпадает с профилем падающего света, угловое расстояние между соседними лучами равно $\Delta \Theta=\lambda / v_{s} T=\lambda \Delta f / v_{s}$. Рис. 5 показывает угловую картину дифракционного поля в зависимости от степени наложения субпорядков, выраженной через параметр $\Delta \Theta / \theta_{0}=\pi r_{0} \Delta f / v_{s}$.

Вследствие наложения порядков картина модулируется с частотами порядка сотен $\mathrm{kHz}$, при этом для каждого угла интенсивность периодически меняется в определенных пределах. В тех приложениях, где время необходимого воздействия в разы превосходит период модуляции, существенным оказывается усредненная картина (сплошная линия). Видно, как при уменьшении интервала $\Delta f$ практически разделенные лучи $(b)$ сближаются $(c)$, формируя в конце концов усредненное распределение с резкими границами $(d)$. При этом диапазон мгновенных значений интенсивности существенно расширяется. Режим $(b)$ используется для многолучевой лазерной записи изображений, в системах переключений оптических каналов и т.д., режим $(d)-$ для создания АО шейпера в данной работе.

\section{2. Поляризационно-нечувствительный дефлектор}

Вторым принципиальным моментом является разработанная трехкристальная схема высокоэффективно- го поляризационно-нечувствительного двухкоординатного АО дефлектора (АОД) [27].

Каждый из трех кристаллов является однокоординатным АОД на базе парателлурита $\left(\mathrm{TeO}_{2}\right)$. Использование АОД - углового сканера света - связано с тем, что брэгговский синхронизм должен выполняться в максимальном угловом диапазоне отклоненного луча. Применение парателлурита обусловлено феноменально большой величиной АО качества $-M_{2} \approx 1000 \cdot 10^{-18} \mathrm{~s}^{3} / \mathrm{g}$ (дифракция на медленной сдвиговой акустической моде), широким диапазоном прозрачности $0.35-5 \mu \mathrm{m}$, высокой лучевой стойкостью, развитой технологией изготовления.

Для высокоэффективной дифракции в $\mathrm{TeO}_{2}$ входной свет должен иметь строго определенную (круговую, либо линейную) входную поляризацию. В то же время большинство мощных промышленных лазеров не обладают таким свойством. Известны методы и схемы создания поляризационно-независимых однокоординатных дефлекторов (АОД) на $\mathrm{TeO}_{2}$, основанные на использовании двух последовательно включенных дефлекторов с фазовой полуволновой пластиной между ними. Очевидная схема двухкоординатного дефлектора заключается в последовательном ортогональном расположении двух однокоординатных дефлекторов и, следовательно, четырех кристаллов $\mathrm{TeO}_{2}$ и двух фазовых пластин, что, очевидно, достаточно сложно.

Принцип работы разработанного устройства, не имеющего фазовых пластин, схематично показан на рис. 6.

Дефлектор содержит три последовательно расположенных кристалла: $C_{1}, C_{2}$ и $C_{3}$. Исходный лазерный луч 0 направлен по оси $Z$ и может быть представлен как суперпозиция двух линейно поляризованных лучей. Первый из них, поляризованный вдоль оси $Y$, не испытывает дифракции и проходит кристалл $C_{1}$ без отклонения - луч 1 . Второй, с поляризацией вдоль оси $X$, претерпевает дифракцию в кристалле $C_{1}$, меняет поляризацию на ортогональную (т.е., вдоль оси $Y$ ) и отклоняется в плоскости $X Z$ на угол, пропорциональный частоте акустической волны - луч 2 . Лучи 1 и 2 одинаково поляризованы и распространяются под небольшим углом друг к другу. Оба луча падают на второй кристалл $C_{2}$ под углом Брэгга, совместно отклоняются в плоскости $Y Z$ на одинаковый угол, и меняют поляризацию на ортогональную, образуя лучи 3 и 4. Кристалл $C_{3}$ ориентирован так, что луч 4 не дифрагирует, а луч 3 испытывает брэгговскую дифракцию и меняет поляризационную моду - луч 5. Если частоты акустических волн в кристаллах $C_{1}$ и $C_{3}$ одинаковы, то выходные лучи 5 и 6 параллельны друг другу.

Таким образом, исходный произвольно поляризованный луч 0 после прохождения трехкристалльного дефлектора преобразуется в совокупность двух параллельных лучей, имеющих ортогональные поляризации, и немного сдвинутых друг относительно друга. Звуковые частоты задают угловое отклонение $\Theta_{x}$ и $\Theta_{y}$ составного 


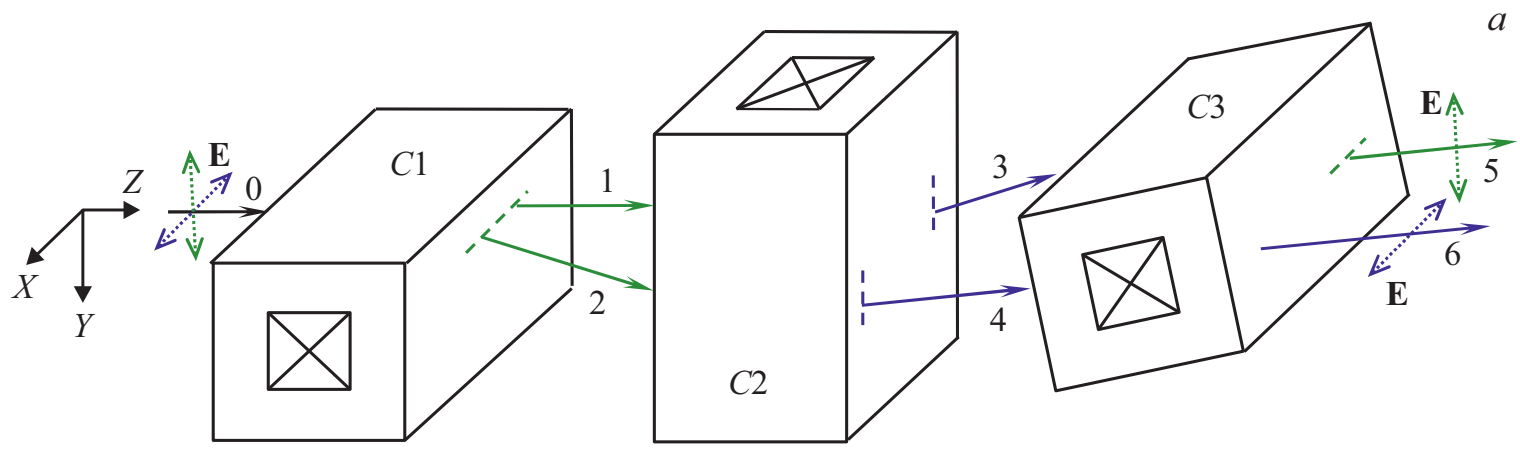

$a$

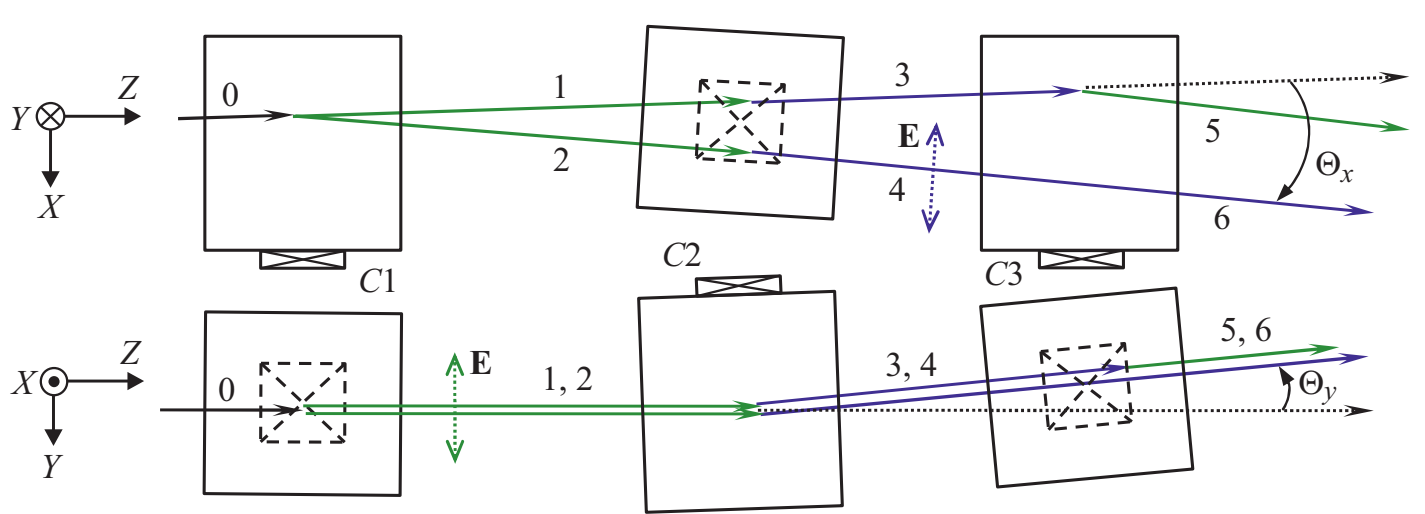

$b$

Рис. 6. $a$ - объемная схема дефлектора, $b-$ ход лучей в ортогональных проекциях. Изображение лучей, как имеющих различное пространственное положение - условное.

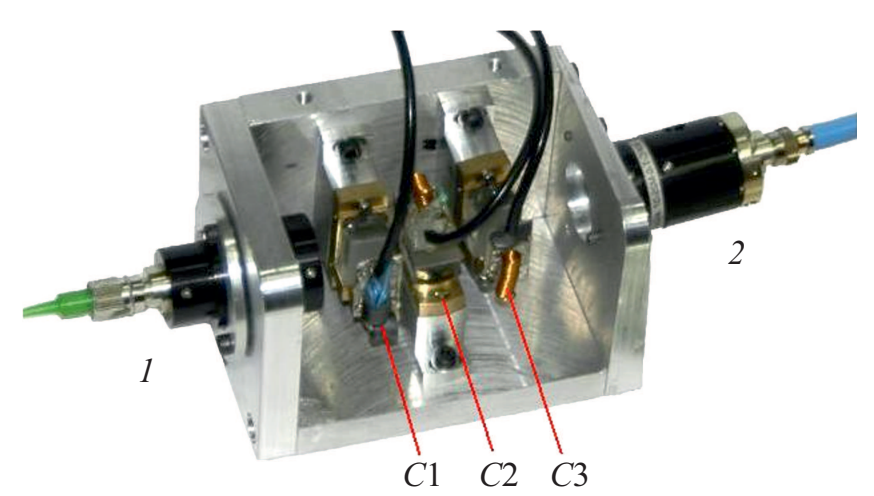

Рис. 7. Внешний вид изготовленного дефлектора. $C_{1}, C_{2}$ и $C_{3}$ - единичные дефлекторы на $\mathrm{TeO}_{2}, 1-$ входной волоконно-оптический коллиматор, $2-$ выходной. Габариты: $100 \times 65 \times 65 \mathrm{~mm}$.

выходного луча в некотором диапазоне, а управляющие напряжения определяют его мощность.

Разработанная конструкция трехкристального АОД показана на рис. 7.

Использовались три идентичных дефлектора с пьезопребразователями из $\mathrm{LiNbO}_{3}$. Размер преобразователя: $L=5 \mathrm{~mm}$ (длина взаимодействия), $H=6 \mathrm{~mm}$ (высота). Акустическая полоса преобразователя 20-50 MHz, длина волны света $\lambda=1.06 \mu \mathrm{m}$. Дефлектор имеет на входе разъем типа $\mathrm{FC}-\mathrm{PC}$.

\section{2. Экспериментальные результаты}

Схема экспериментальной установки для $2 D$-модификации энергетического профиля лазерного излучения приведена на рис. 8.

Следует отметить, что предложенная схема имеет ограничение по форме реализуемого двумерного профиля. Радиосигналы, поданные на первый и второй дефлекторы, формируют независимые одномерные профили $I_{1}(x)$ и $I_{2}(y)$. Итоговая картина является их произведением: $I(x, y)=I_{1}(x) I_{2}(y)$. В частности, таким методом невозможно сформировать аксиальносимметричный профиль.

В экспериментах использовался волоконный лазер (без выделенного направления вектора поляризации) с качеством луча $M^{2}<1.1$, что практически означает гауссов профиль излучения. Электронная система управления генерировала пять эквидистантных радиочастот: центральная - $41 \mathrm{MHz}$ и четыре симметрично расположенные боковые $\pm 0.3, \pm 0.6 \mathrm{MHz}$. Исходно выбирались такие амплитуды и фазы частотных компонент, которые грубо реализуют необходимый профиль при условии минимальной амплитудной модуляции радиосигнала. Затем, обрабатывая дифракционную картину с помощью вычислительной техники, проводилось итерационное уточнение параметров. Критерием являлось получение заданного двумерного профиля при максимальной эф- 


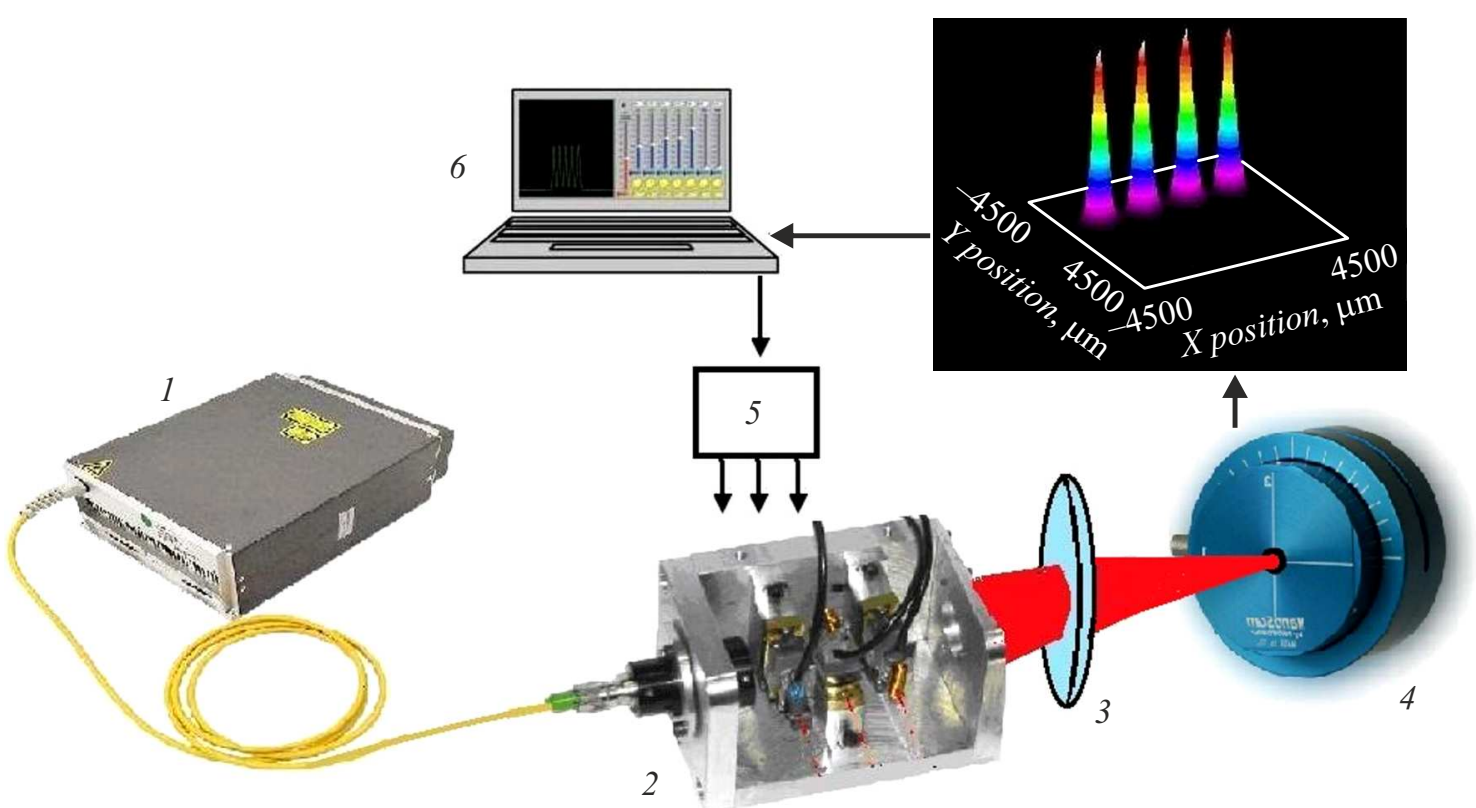

Рис. 8. Схема установки: $1-$ волоконный лазер с длиной волны $\lambda=1.06 \mu \mathrm{m}, 2-$ АОД, $3-$ формирующая оптическая система, 4 - измеритель профиля лазерного луча модели ВР104, 5 - трехканальный синтезатор частот, 6 - компьютер.

$a$

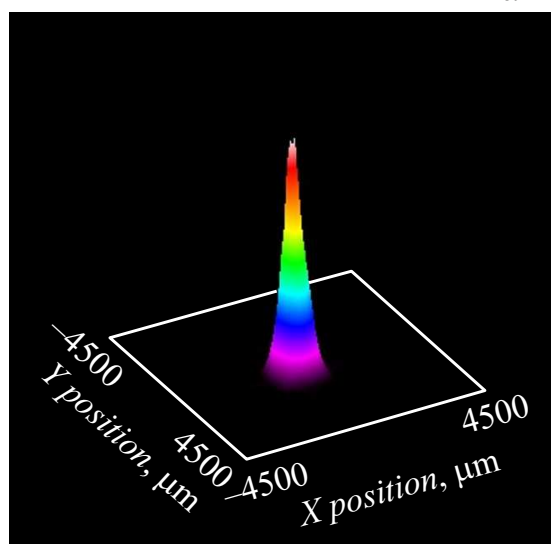

$b$

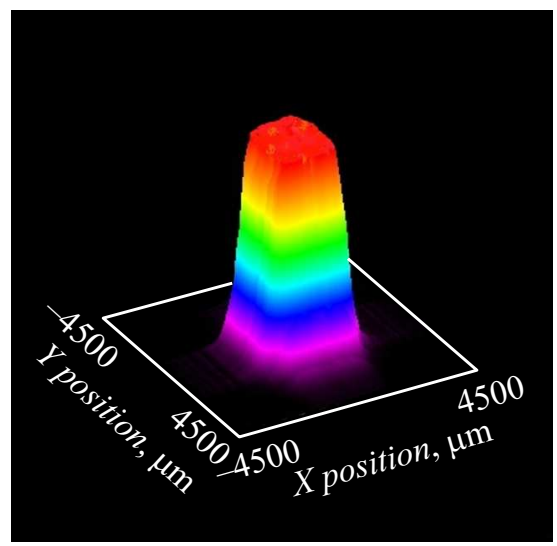

c

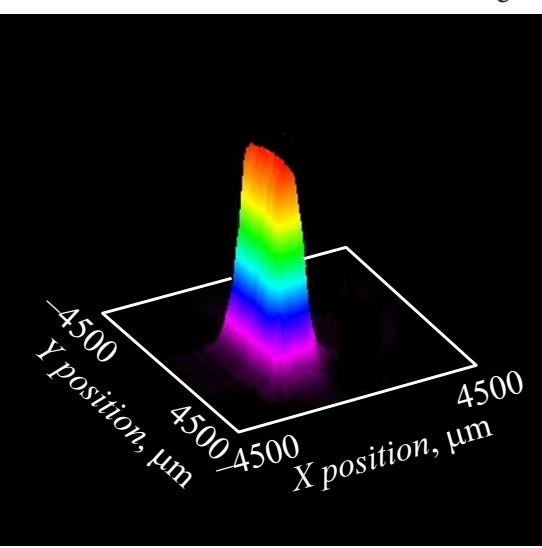

Рис. 9. $2 D$-профили исходного $(a)$ и дифрагированного $(b, c)$ поля на выходе АОД. $b$ - симметричный по $X$ - и $Y$-направлениям профиль, $c$ - несимметричный профиль.

фективности дифракции. Измеритель профиля отражал пространственное $2 D$-распределение интенсивности дифрагированного поля. Кроме того, измеритель профиля позволял производить абсолютные измерения мощности поступающего оптического излучения. Тем самым эффективность дифракции (преобразование профиля) измерялась как отношение суммарной мощности преобразованного луча к мощности прошедшего излучения (без включения дефлекторов). Достигнутая эффективность составила 85-90\% (в зависимости от формы и ширины итогового профиля).

Экспериментально полученные $2 D$-профили приведены на серии рис. 9 (для каждого рисунка масштаб по мощности нормирован к единице).
Таким образом, использование выбранного режима многолучевой дифракции вместе с трехкристальным поляризационно-независимым АОД позволяет эффективно трансформировать энергетический профиль лазерного излучения независимо от характера его поляризации.

В настоящей работе использовался лазер, мощность которого равна $20 \mathrm{~W}$. С учетом опыта предыдущих исследований можно с уверенностью сказать, что кристаллы $\mathrm{TeO}_{2}$ хорошего качества (материала и оптических поверхностей) бездефектно работают с плотностями мощностей до сотен $\mathrm{kW} / \mathrm{cm}^{2}$.

Практическое использование результатов работы в настоящее время изучается применительно к системам лазерной гравировки. 


\section{Заключение}

1. Разработан акустооптический двухкоординатный метод изменения поперечного распределения интенсивности лазерного излучения. В основе лежит деление лазерного луча на совокупность частично перекрывающихся в угловом пространстве лучей, интенсивность и положение которых задается управляющим радиосигналом.

2. Рассмотренный метод имеет следующие особенности. Во-первых, из-за частотного сдвига между лучами дифракционная картина не является стационарной, и требуемый профиль реализуется при усреднении во времени. Во-вторых, двумерный профиль интенсивности реализуется только в виде произведения двух независимых одномерных профилей. В-третьих, возможна быстрая смена профилей, что определяется временем пересечения апертуры света звуковой волной в кристалле.

3. Метод реализован экспериментально на базе трехкристального высокоэффективного поляризационнонезависимого двухкоординатного АО дефлектора. Для лазерного излучения с длиной волны $1.06 \mu \mathrm{m}$ получены несколько профилей (в частности, близкий к равномерному) при суммарной эффективности не менее чем $85 \%$. Время смены профиля составило около $10 \mu \mathrm{s}$.

\section{Финансирование работы}

Работа выполнена за счет бюджетного финансирования в рамках государственного задания по теме 00302019-0014.

\section{Благодарности}

Авторы благодарят Благотворительный Фонд Андрея Мельниченко (The Andrey Melnichenko Foundation) за помощь при проведении данного исследования.

\section{Конфликт интересов}

Авторы заявляют, что у них нет конфликта интересов.

\section{Список литературы}

[1] А.Г. Григорьянц. Основы лазерной обработки материалов (Машиностроение, М., 1989)

[2] E. Kannatey-Asibu. Principles of Laser Materials Processing (Wiley, Hoboken, 2009)

[3] W.M. Steen, J. Mazumder. Laser Material Processing (Springer, NY., 2010). DOI: 10.1007/978-1-84996-062-5

[4] Г.Г. Гладуш, И. Ю. Смуров. Физические основы лазерной обработки материалов (Физматлит, М., 2017)

[5] F.M. Dickey, S.C. Holswade. Laser Beam Shaping: Theory and Techniques (Marcel Dekker, NY., 2000)
[6] Д.Л. Головашкин, Л.Л. Досколович, Н.Л. Казанский, В.В. Котляр, В.С. Павельев, Р.В. Скиданов, В.А. Сойфер, С.Н. Хонина. Дифракционная компьютерная оптика / ред. В.А. Сойфер. (Физматлит, М., 2007). [Computer Design of Diffractive Optics. Ed. by V.A. Soifer. (International Science Publishing Ltd. \& Woodhead Pub. Ltd., Cambridge, 2012)

[7] K. Fuse. LTJ, 12 (2), 19 (2015). DOI: 10.1002/latj.201500011

[8] A. Laskin, V. Laskin. J. Phys.: Conf. Ser. 276, 12171 (2011). DOI: 10.1088/1742-6596/276/1/012171

[9] Л.Н. Магдич, В.Я. Молчанов. Акустооптические устройства и их применение (Сов. радио, М., 1978). [L.N. Magdich, V.Ya. Molchanov. Acoustooptic Devices and Their Applications (Gordon and Breach, NY., 1989)]

[10] J. Sapriel. Acousto-Optics (Wiley, NY., 1979)

[11] В.И. Балакший, В.Н. Парыгин, Л.Е. Чирков. Физические основы акустооптики (Радио и связь, М., 1985)

[12] А. Корпел. Акустооптика (Мир, М., 1993). [A. Korpel. Acousto-optics (Marcel Dekker, NY., 1988)]

[13] J. Xu, R. Stroud. Acousto-optic devices (Wiley, NY., 1992)

[14] А.С. Задорин. Динамика акустооптического взаимодействия (ТГУ, Томск, 2004)

[15] В.Я. Молчанов, Ю.И. Китаев, А.И. Колесников, В.Н. Нарвер, А.3. Розенштейн, Н.П. Солодовников, К.Г. Шаповаленко. Теория и практика современной акустооптики (МИСиС, М., 2015)

[16] M. McNeill, Ting-Chung. Poon. Appl. Opt., 33 (20), 4508 (1994). DOI: 10.1364/AO.33.004508

[17] W. Akemann, J.-F. Léger, C. Ventalon, B. Mathieu, S. Dieudonné, L. Bourdieu. Opt. Express, 23 (22), 28191 (2015). DOI: 10.1364/OE.23.028191

[18] J. Strauß, M. Vorndran, J. Heberle, M. Schmidt. ICALEO 2016, M1002 (2016). DOI: 10.2351/1.5118603

[19] P. Bechtold, R. Hohenstein, M. Schmidt. Opt. Express, 21 (12), 14627 (2013). DOI: 10.1364/OE.21.014627

[20] С.Н. Антонов, О.А. Бышевский-Конопко, А.В. Вайнер, В.В. Проклов, Ю.Г. Резвов. ЖТФ, 78 (5), 78 (2008). [S.N. Antonov, O.A. Byshevskii-Konopko, A.V. Vainer, V.V. Proklov, Yu.G. Rezvov. Tech. Phys, 53 (5), 609 (2008). DOI: $10.1134 /$ S1063784208050137]

[21] K.B. Yushkov, V.Ya. Molchanov, V.I. Balakshy, S.N. Mantsevich. Proc. SPIE, 10744, 107440Q (2018). DOI: $10.1117 / 12.2315329$

[22] K.B. Yushkov, A.I. Chizhikov, O.Yu. Makarov, V.Ya. Molchanov. Appl. Opt., 59(28), 8575 (2020). DOI: $10.1364 / A O .398626$

[23] D. Trypogeorgos, T. Harte, A. Bonnin, C. Foot. Opt. Express, 21 (21), 24837 (2013). DOI: 10.1364/OE.21.024837

[24] С.Н. Антонов, А.Л. Филатов. ЖТФ, 88 (1), 93 (2018). DOI: $10.21883 /$ JTF.2018.01.45489.2266 [S.N. Antonov, A.L. Filatov. Tech. Phys., $63(1), 90$. DOI: $10.1134 / \mathrm{S} 1063784218010048]$

[25] С.Н. Антонов, А.В. Вайнер, В.В. Проклов, Ю.Г. Резвов. ЖТФ, $78(6), 79$ (2008). [S.N. Antonov, A.V. Vainer, V.V. Proklov, Y.G. Rezvov. Tech. Phys., 53 (6), 752 (2008). DOI: 10.1134/S1063784208060145]

[26] S.N. Antonov, A.V. Vainer, V.V. Proklov, Y.G. Rezvov. Appl. Opt., 48 (7), C171 (2009). DOI: 10.1364/AO.48.00C171

[27] С.Н. Антонов. ЖТФ, 89 (2), 274 (2019). DOI: 10.21883/JTF. 2019.02.47083.205-18 [S.N. Antonov. Tech. Phys., 64 (2), 242 (2019). DOI: 10.1134/S1063784219020038] 